\title{
Composición, Distribución y Abundancia del Ictioplancton en la Cuenca del Océano Pacífico Colombiano durante Septiembre de 2003
}

\author{
Eugenia Escarria Gómez ${ }^{1}$ \\ Beatriz Susana Beltrán-León ${ }^{2}$ \\ Alan Giraldo López ${ }^{3}$ \\ Javier Roberto Ortiz Galvis ${ }^{4}$ \\ 1-3 Departamento de Biología. Grupo de Investigación en Ecología Animal. Universidad del Valle \\ A.A. 25360 Cali, Valle, Colombia \\ 1e-mail: euesgo79@yahoo.com \\ 3e-mail: agiraldo@univalle.edu.co
}

\author{
${ }^{2}$ Dirrección Territorial Suroccidental. Unidad Administrativa Especial del Sistema de Parques Nacionales Naturales, - \\ UAESPNN. \\ Av. 3GN No. 37N 70, Cali, Valle, Colombia \\ e-mail: mmambiente@emcali.net.co \\ e-mail: bbeltranleon@yahoo.com \\ ${ }^{4}$ Área de Oceanografía Operacional, Centro Control Contaminación del Pacífico - CCCP \\ Vía El Morro, Capitanía de Puerto, San Andrés de Tumaco, Nariño, Colombia \\ Tél: +57 (2) 72726 37, Fax: +57 (2) 7271180 , AA. 187 \\ e-mail: cccp.webmaster@dimarnet.mil.co \\ e-mail: jortiz@dimarnet.mil.co
}

Escarria, E., B. Beltrán-León, A. Giraldo y J. Ortiz. 2005. Composición, Distribución y Abundancia del Ictioplancton en la Cuenca del Océano Pacífico Colombiano durante Septiembre de 2003. Boletín Científico CCCP, (12): 23-35.

Recibido en septiembre de 2004; aceptado en octubre de 2005

RESUMEN

Este trabajo presenta resultados de los análisis del ictioplancton obtenidos mediante arrastres superficiales en 23 estaciones de muestreo durante la campaña oceanográfica Pacífico XXXVIII ERFEN - XXXVI en la cuenca del océano Pacífico colombiano, CPC. Se identificaron los estadios larvales de 19 especies pertenecientes a 18 familias, en su mayoría de interés para la pesca. Se destacó por su abundancia la familia Photichthyidae, seguida de la familia Hemiramphidae, que presentó la más amplia distribución. Se determinó la distribución y abundancia de huevos mediante el análisis gráfico de mapas de dispersión espacial y se evaluó la relación entre la distribución y abundancia de larvas de peces con la temperatura, salinidad, oxígeno, clorofila-a y variación nictimeral. El comportamiento de las variables físico-químicas no evidenciaron cambios anómalos, considerándose éste período como normal. No se detectaron diferencias significativas para la abundancia del ictioplancton entre el día y la noche (Mann Whitney, $\mathrm{P}=0.2974)$. Sin embargo, la distribución y abundancia de las larvas en la zona costera y oceánica estuvo influenciada por la clorofila- $a$, la temperatura y la salinidad. Teniendo en cuenta el error de muestreo asumido por el uso de una red cónica simple y el tipo de arrastre, se considera que la abundancia de las larvas de peces fue subestimada.

Palabras clave: Ictioplancton, huevos de peces, larvas de peces, Pacífico colombiano, costero, oceánico.

Abstract

The results of the analysis of the icthyoplankton obtained by means of superficial trawling in 23 sampling stations during the campaign Pacific XXXVIII ERFEN - XXXVI in the Colombian Pacific basin, are presented. The larval stages of 19 species belonging to 18 families were identified, most of them considered commercially valuable. The family Photichthyidae stands out for its abundance, followed by the family Hemiramphidae which showed the widest distribution. The eggs distribution and abundance was determined by means of the graphical analysis of spatial dispersion maps and the relationship between the fish larval distribution and abundance with the temperature, salinity, oxygen, chlorophyll a and nictimeral variation was evaluated. The physical-chemical variables behavior did not show anomalous changes, consequently it is considered as a normal period. There was no significant differences between the icthyoplankton abundance during day and night (Mann Whitney, $\mathrm{P}=0.2974$ ). Nevertheless, the larval distribution and abundance in the coastal and oceanic zones was influenced by the chlorophyll, temperature and salinity. Considering the error for the simple conical net use and the type of trawling, it is suggested that the fish larval abundance is sub-estimated.

KeY wORDs: Icthyoplankton, fish eggs, fish larvae, Colombian Pacific Ocean, coastal, oceanic. 


\section{INTRODUCCIÓN}

El ictioplancton es un componente de gran importancia dentro de las comunidades planctónicas. En él se encuentran agrupados los huevos, larvas, post-larvas y algunas veces los juveniles de peces. La aparición de huevos y larvas de distintas especies de peces en el mar está íntimamente relacionada con el ciclo anual de la maduración sexual de los adultos. Por lo tanto su composición cualitativa y cuantitativa varía, generalmente, en escalas anuales y está sujeta al efecto directo de distintos procesos fisiológicos de los peces adultos (Ciechomski, 1981).

El ictioplancton sugiere, a través de la variabilidad en su composición y abundancia, la calidad o el deterioro de los medios que ocupa. Económicamente, la importancia de las investigaciones del ictioplancton radica en que proporcionan el conocimiento necesario para comprender muchos aspectos de las pesquerías encaminados hacia un manejo racional. Por ejemplo, los estudios sobre la mortalidad de huevos y larvas pueden proveer la clave para entender la relación 'stock-reclutamiento' con más razón, si se tiene en cuenta que tan sólo un $10 \%$ o menos del total de los huevos desovados constituirán el número de individuos que se recluten al stock (Beltrán-León y Ríos, 2000).

Los stocks de peces y sus potenciales de explotación son evaluados mediante investigaciones sistemáticas que requieren, necesariamente, el estudio de huevos y larvas. Específicamente, aspectos relacionados con su abundancia y distribución en relación con parámetros fisicoquímicos y biológicos lo convierte en una herramienta-fundamental en las evaluaciones pesqueras (Rueda-Montenegro y Beltrán, 1992). En este sentido, la evaluación del ictioplancton tiene aplicaciones directas en el manejo y control de las pesquerías, al servir como base para la determinación de zonas y épocas de desove; biomasa de adultos efectivos desovantes; migración de las poblaciones; relaciones entre las condiciones oceanográficas y la distribución espaciotemporal, y abundancia de larvas y adultos.

El estudio del ictioplancton en el Pacífico colombiano comenzó con los trabajos realizados en la bahía de Buenaventura por Echeverri y Bergamín
(1982), López-Peralta (1984) y Rueda-Montenegro y Caraballo (1984). Sin embargo, a principio de los años 90, una aproximación holística y sistemática para el estudio del ictioplancton permitió ampliar la escala espacial de muestreo (Jiménez, 1991). En este mismo sentido, el Programa Regional de Cooperación Técnica para la Pesca, CEEPEC/INDERENA/INPA ALA $87 / 21$, patrocinó tres cruceros de evaluación íctica desde la frontera con Panamá hasta la frontera con Ecuador y desde la línea de costa hasta las 40 millas náuticas, incrementando considerablemente el conocimiento previo de la comunidad ictioplanctónica del Pacífico colombiano (BeltránLeón, 1992; Rueda-Montenegro y Beltrán-León, 1992; Beltrán-León et al., 1994; Moreno, 1995). Uno de los estudios más completos sobre el ictioplancton de la CPC fue el realizado por Beltrán-León y Ríos (2000). Estos autores estudiaron la taxonomía de larvas y postlarvas de peces pertenecientes a 21 órdenes, 89 familias, 180 géneros y 236 especies; complementándola con las características de abundancia, hábitat y biología.

Con este trabajo se pretende contribuir al conocimiento de la composición, distribución y abundancia de larvas y huevos de peces de la CPC durante septiembre 2003 y su relación con factores físicos y químicos.

\section{Área de Estudio}

El estudio se realizó en el área comprendida entre los meridianos $77^{\circ} 45^{\prime} 0$ a $84^{\circ} 00^{\prime} \mathrm{O}$ y entre las latitudes $1^{\circ} 30^{\prime} \mathrm{N}$ a $6^{\circ} 30^{\prime} \mathrm{N}$ (Figura 1). Cabo Corrientes divide en dos sectores geomorfológicos la zona costera del Pacífico colombiano; al Norte se presenta una costa rocosa con acantilados y plataforma continental estrecha; al Sur la costa es baja y se encuentran numerosas desembocaduras de cortos pero caudalosos ríos, lo que favorece la presencia de grandes depósitos sedimentarios frente a la costa (Montagut y Castillo, 1989). El clima de esta región es de tipo ecuatorial cálido y húmedo, con precipitaciones que oscilan entre 3000 y $5000 \mathrm{~mm}$ año ${ }^{-1}$. La salinidad varía entre 27 , en regiones cercanas a la costa, y 33.5, mar adentro; la temperatura superficial del mar se encuentra en un rango entre 26.5 a $27^{\circ} \mathrm{C}$ (CCCP, 2003). 


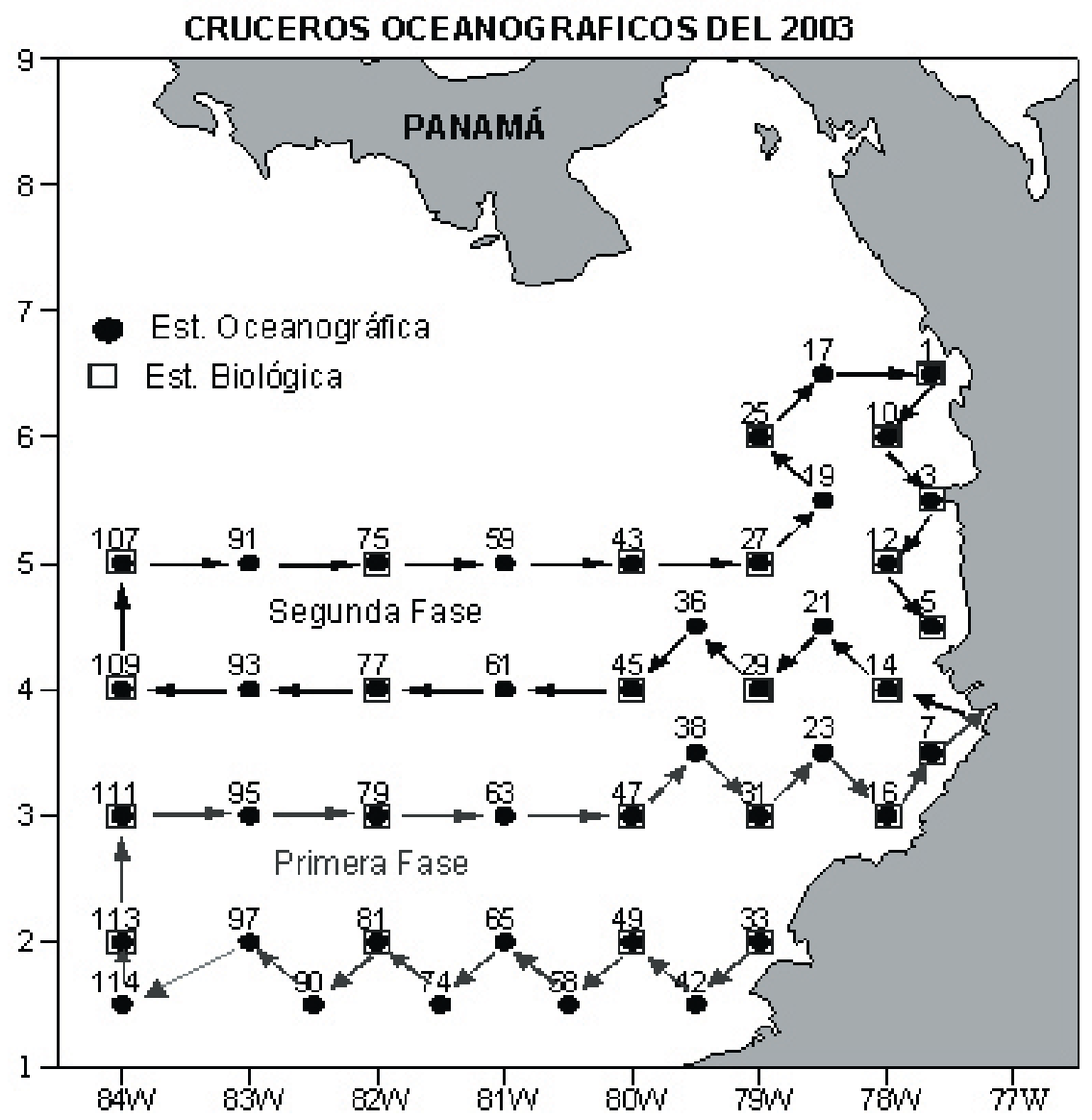

Figura 1. Grilla de estaciones realizadas en el crucero oceanográfico PACíFICO XXXVIII- ERFEN XXXVI septiembre 2003 en el Pacífico colombiano. Fuente: CCCP, 2003.

\section{Metodología}

En la campaña oceanográfica Pacífico XXXVIII - ERFEN XXXVI, a bordo del buque oceanográfico ARC MALPELO, se realizaron pescas de zooplancton y el registro de parámetros físico-químicos de temperatura, salinidad, oxígeno disuelto y concentración de clorofila- $a$, siguiendo una grilla de muestreo de 44 estaciones equiespaciadas en la CPC (Figura 1).

Los arrastres de zooplancton se realizaron en 25 estaciones de la grilla establecida (Tabla I), con excepción de las estaciones 43 y 75 , en las cuales se presentaron problemas técnicos. Para la captura de zooplancton se utilizó una red cónica simple con orificio de malla de 363 micras, provista de un flujómetro digital. Las capturas de zooplancton se realizaron mediante arrastres superficiales de 10 minutos a una velocidad de 3 nudos. El zooplancton colectado durante los arrastres fue preservado en frascos transparentes de $1 \mathrm{~L}$, con formol buferizado al $10 \%$ en agua de mar para ser transportado al laboratorio, donde se separaron las larvas y huevos de peces de la muestra de zooplancton, utilizando un microscopio estereoscopio, y se preservaron en formol al $5 \%$ neutralizado con borax. La identificación de las larvas de peces se realizó con base en los caracteres merísticos, morfométricos y de pigmentación, hasta el menor nivel taxonómico posible (familia, género y/o especie), siguiendo las claves de identificación de: Ahlstrom (1983), Ciechomski (1981), Moreno (1995), Moser (1996) y Beltrán-León y Ríos (2000). 
Tabla I. Posición geográfica de las estaciones de muestreo y su división longitudinal durante la campaña oceanográfica Pacífico XXXVIII ERFEN - XXXVI, realizada en la CPC durante septiembre de 2003.

\begin{tabular}{|c|c|c|c|}
\hline Estación & \multicolumn{2}{|c|}{ Localización } & \multirow[t]{2}{*}{ Zona } \\
\hline & latitud N & / longitud & \\
\hline 1 & $06^{\circ} 03^{\prime} 00^{\prime \prime}$ & $77^{\circ} 45^{\prime} 00^{\prime}$ & Costera \\
\hline 3 & $05^{\circ} 03^{\prime} 00^{\prime}$ & $77^{\circ} 45^{\prime} 00^{\prime}$ & Costera \\
\hline 5 & $04^{\circ} 30^{\prime} 00^{\prime \prime}$ & $77^{\circ} 45^{\prime} 00^{\prime}$ & Costera \\
\hline 7 & $03^{\circ} 30^{\prime} 00^{\prime \prime}$ & $77^{\circ} 45^{\prime} 00^{\prime}$ & Costera \\
\hline 10 & $06^{\circ} 00^{\prime} 00^{\prime \prime}$ & $78^{\circ} 00^{\prime} 00^{\prime \prime}$ & Costera \\
\hline 12 & $05^{\circ} 00^{\prime} 00^{\prime \prime}$ & $78^{\circ} 00^{\prime} 00^{\prime \prime}$ & Costera \\
\hline 14 & $04^{\circ} 00^{\prime} 00^{\prime \prime}$ & $78^{\circ} 00^{\prime} 00^{\prime \prime}$ & Costera \\
\hline 16 & $03^{\circ} 00^{\prime} 00^{\prime \prime}$ & $78^{\circ} 00^{\prime} 00^{\prime \prime}$ & Costera \\
\hline 25 & $06^{\circ} 00^{\prime} 00^{\prime \prime}$ & $79^{\circ} 00^{\prime} 00^{\prime \prime}$ & Costera \\
\hline 27 & $05^{\circ} 00^{\prime} 00^{\prime}$ & $79^{\circ} 00^{\prime} 00^{\prime}$ & Costera \\
\hline 29 & $04^{\circ} 00^{\prime} 00^{\prime \prime}$ & $79^{\circ} 00^{\prime} 00^{\prime}$ & Costera \\
\hline 31 & $03^{\circ} 00^{\prime} 00^{\prime \prime}$ & $79^{\circ} 00^{\prime} 00^{\prime}$ & Costera \\
\hline 33 & $02^{\circ} 00^{\prime} 00^{\prime \prime}$ & $79^{\circ} 00^{\prime} 00^{\prime}$ & Costera \\
\hline 45 & $04^{\circ} 00^{\prime} 00^{\prime \prime}$ & $80^{\circ} 00^{\prime} 00^{\prime \prime}$ & Costera \\
\hline 47 & $03^{\circ} 00^{\prime} 00^{\prime \prime}$ & $80^{\circ} 00^{\prime} 00^{\prime}$ & Costera \\
\hline 49 & $02^{\circ} 00^{\prime} 00^{\prime \prime}$ & $80^{\circ} 00^{\prime} 00^{\prime \prime}$ & Costera \\
\hline 77 & $04^{\circ} 00^{\prime} 00^{\prime \prime}$ & $82^{\circ} 00^{\prime} 00^{\prime \prime}$ & Oceánica \\
\hline 79 & $03^{\circ} 00^{\prime} 00^{\prime \prime}$ & $82^{\circ} 00^{\prime} 00^{\prime}$ & Oceánica \\
\hline 81 & $02^{\circ} 00^{\prime} 00^{\prime \prime}$ & $82^{\circ} 00^{\prime} 00^{\prime}$ & Oceánica \\
\hline 107 & $05^{\circ} 00^{\prime} 00^{\prime \prime}$ & $84^{\circ} 00^{\prime} 00^{\prime}$ & Oceánica \\
\hline 109 & $04^{\circ} 00^{\prime} 00^{\prime \prime}$ & $84^{\circ} 00^{\prime} 00^{\prime}$ & Oceánica \\
\hline 111 & $03^{\circ} 00^{\prime} 00^{\prime \prime}$ & $84^{\circ} 00^{\prime} 00^{\prime \prime}$ & Oceánica \\
\hline 113 & $02^{\circ} 00^{\prime} 00^{\prime \prime}$ & $84^{\circ} 00^{\prime} 00^{\prime \prime}$ & Oceánica \\
\hline
\end{tabular}

Se registró la abundancia de cada especie estandarizando los datos del recuento a ind $1000 \mathrm{~m}^{-3}$ y se estimó la biomasa humedad del zooplancton estandarizada a $\mathrm{ml} 1000 \mathrm{~m}^{-3}$. En las estaciones de muestreo se registraron parámetros físico-químicos de salinidad y temperatura utilizando un perfilador de conductividad, temperatura y profundidad CTD
SBE-19. La temperatura superficial de mar, TSM, se registró directamente con un termómetro de cazoleta. Se estimó la concentración de oxígeno disuelto y la concentración de clorofila- $a$, a profundidades estándar, mediante un muestreo discreto con botellas Niskin. La concentración de oxígeno se estableció siguiendo el método de titulación de Winkler, mientras que la concentración de clorofila- $a$ se precisó siguiendo el método espectofotométrico estándar a partir de la extracción en acetona al $90 \%$.

La dispersión espacial de las familias de larvas de peces se representó en mapas, de acuerdo con el rango de abundancia utilizado por RuedaMontenegro y Beltrán-León (1992). Se analizó gráficamente la dispersión de la temperatura, salinidad y oxígeno superficial en la zona de estudio, utilizando el método de interpolación espacial Krigging. Para realizar el análisis estadístico se dividió el área de estudio en zona costera y oceánica, considerando el ancho de la plataforma continental. Con base en este criterio se definieron las estaciones de muestreo que se encontraron entre los $77^{\circ} 45^{\prime} 0$ hasta los $79^{\circ} 02^{\prime} 0$ y desde los $01^{\circ} 30^{\prime} \mathrm{N}$ hasta los $06^{\circ} 30^{\prime} \mathrm{N}$ como costeras, mientras que las estaciones que se encontraron entre los $80^{\circ} 00^{\prime} 0$ hasta los $84^{\circ} 00^{\prime} \mathrm{O}$ y desde los $01^{\circ} 30^{\prime} \mathrm{N}$ hasta los $05^{\circ} 00^{\prime} \mathrm{N}$ fueron consideradas oceánicas.

Se evaluaron las diferencias temporales de abundancia en una escala Diel, utilizando la prueba no paramétrica de Mann-Whitney. En este mismo sentido, el análisis estadístico de la dinámica espacial de la abundancia de ictioplancton se realizó considerando como variables independientes las condiciones fisicoquímicas y como factores de agregación la zona costera y oceánica.

Se realizó un análisis de correlación de Sperman para evaluar el grado de asociación entre la abundancia de larvas y los parámetros fisicoquímicos, tanto en la zona costera como en la zona oceánica. Todos los análisis estadísticos se realizaron con el programa computacional Statistica 6.0 .

\section{Resultados}

Durante el período de muestreo se registraron diferencias significativas entre la zona costera y oceánica para la TSM; salinidad, SSM, y concentración de clorofila- $a$, Clo-a (Mann-Whitney 
test: TSM p $=0.0004$, SSM p $=0.004$, Clo- $a \mathrm{p}=$ 0.02 ). La TSM en la zona costera fue más alta que en la zona oceánica (promedio TSM costa $=27.55{ }^{\circ} \mathrm{C} /$ promedio $\mathrm{TSM}$ océano $=26.32{ }^{\circ} \mathrm{C}$ ) . La salinidad más alta en el área de estudio se registró en la zona oceánica (promedio SSM costa $=31.5 /$ promedio SSM océano $=33.2$ ) . El análisis de la dispersión espacial de la clorofila- $a$ en la zona de estudio sugiere una alta homogeneidad espacial. Sin embargo, los

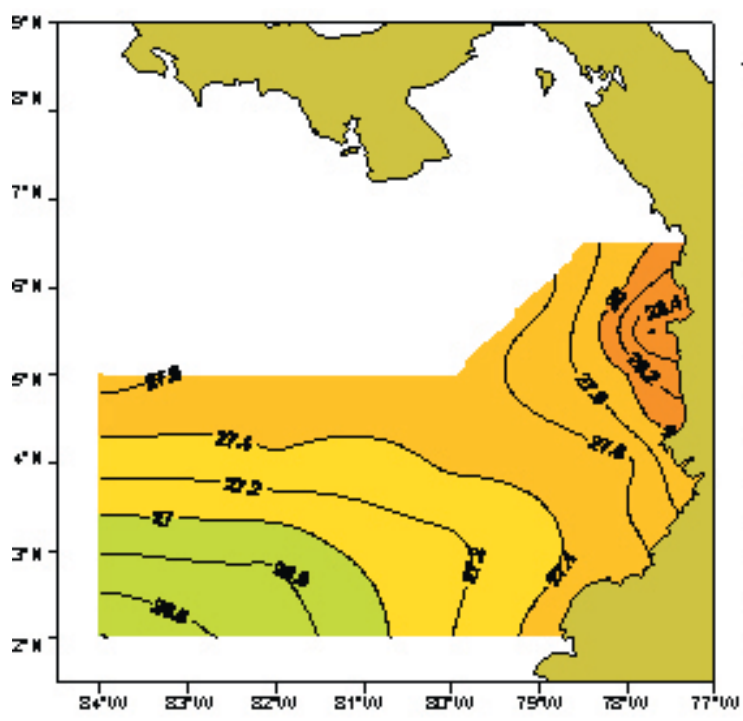

valores estimados en la zona costera son dos órdenes de magnitud mayores que los estimados para la zona oceánica (i.e. Estación 5 clo- $a=1.37 \mathrm{mg} \mathrm{m}^{-3} /$ Estación $113 \mathrm{Clo}-a=$ $0.01 \mathrm{mg} \mathrm{m}^{-3}$ ). Aunque las concentraciones de oxígeno disuelto más altas fueron registradas en la zona costera (Figura 2), se detectaron diferencias para este parámetro entre la zona costera y oceánica (rango de variación entre $\left.4.50 \mathrm{ml} \mathrm{l}^{-1} \mathrm{a} 4.63 \mathrm{ml} \mathrm{l}^{-1}\right)$.

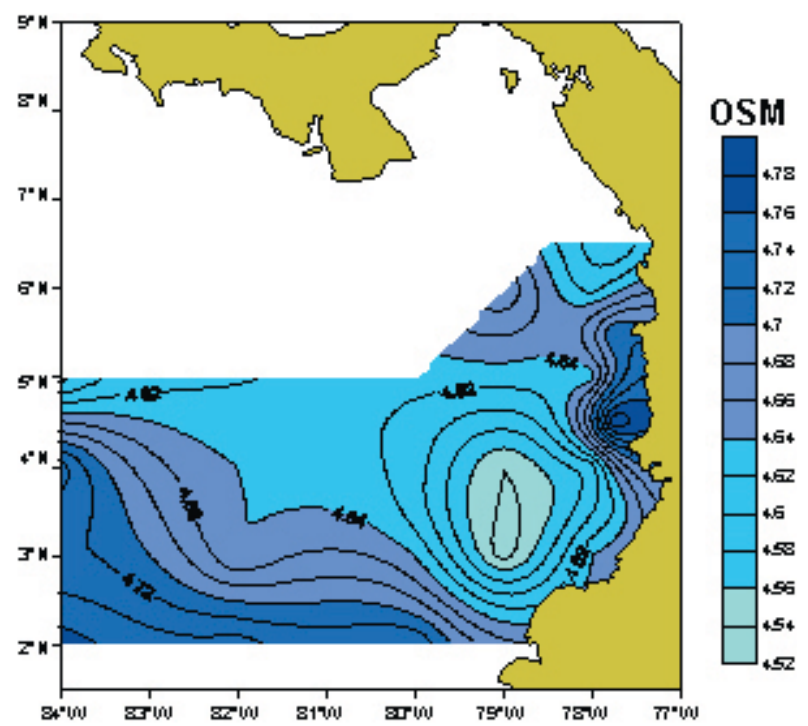

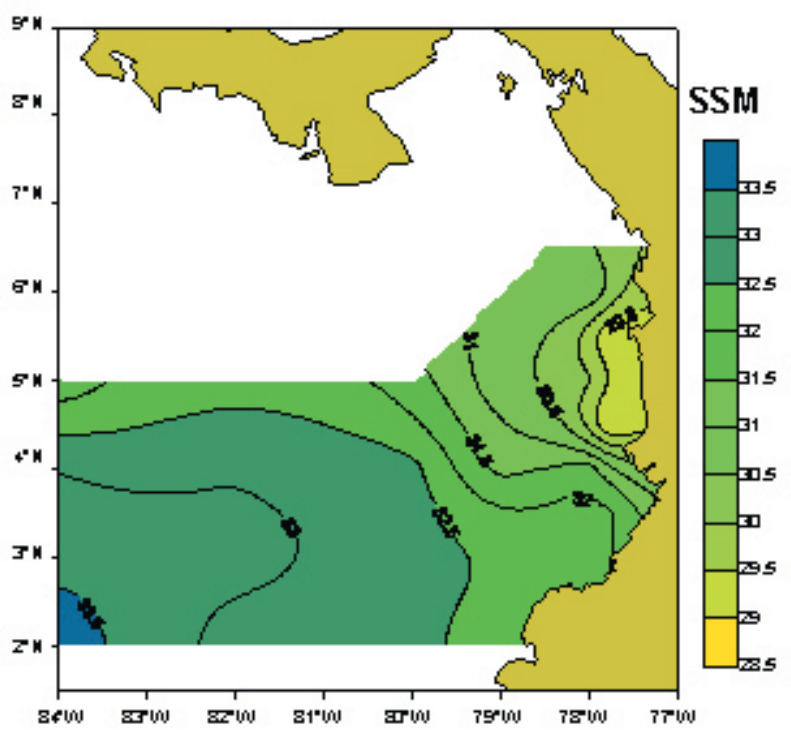

Figura 2. Temperatura superficial del $\operatorname{mar}\left(T S M,{ }^{\circ} \mathrm{C}\right)$, salinidad superficial del $\operatorname{mar}(\mathrm{SSM})$ y oxígeno disuelto superficial del mar (OSM, ml/l), en la CPC, durante septiembre 2003. 
La biomasa zooplanctónica osciló

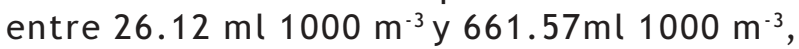
con un valor medio de $232.76 \mathrm{ml} 1000 \mathrm{~m}^{-3}$ (Tabla II). No se detectó un patrón específico de dispersión espacial para la biomasa zooplanctónica en la zona de estudio (Figura 3 ). Todas las estaciones fueron positivas para huevos de peces, siendo contabilizados un total de 24408 huevos $1000 \mathrm{~m}^{-3}$ (Tabla II). El rango de abundancia de huevos de peces estuvo entre 8 y 5687 huevos $1000 \mathrm{~m}^{-3}$, registrándose la menor abundancia de huevos en la zona costera (Figura 3). La abundancia de larvas de peces en el área de estudio estuvo entre 7 a 200 larvas $1000 \mathrm{~m}^{-3}$, registrándose la presencia efectiva en el
$82.60 \%$ de las estaciones muestreadas (Tabla II).

Contrario a lo esperado, no se detectaron diferencias significativas en la abundancia de larvas de peces entre el día y la noche (Mann-Whitney test, $\mathrm{p}=0.2974$ ). En este mismo sentido, al evaluar el grado de asociación entre los parámetros físicoquímico-biológicos y la abundancia de larvas de peces, considerando la zona costera y la zona oceánica en forma conjunta, no se detectó una asociación significativa entre el oxígeno disuelto y la abundancia de larvas; sin embargo, los datos sugieren una posible correlación (Sperman $\mathrm{p}=0.06$ ).

Tabla II. Abundancia de biomasa zooplanctónica, huevos y larvas de peces en cada una de las estaciones de muestreo.

\begin{tabular}{|c|c|c|c|}
\hline Estación & $\begin{array}{c}\text { Biomasa zoo } \\
\mathrm{ml} 1000 \mathrm{~m}^{-3}\end{array}$ & $\begin{array}{l}\text { Abundancia huevos } \\
\qquad 1000 \mathrm{~m}^{-3}\end{array}$ & $\begin{array}{c}\text { Abundancia larvas } \\
1000 \mathrm{~m}^{-3}\end{array}$ \\
\hline 1 & 84.41 & 8 & 25 \\
\hline 3 & 218 & 21 & 22 \\
\hline 5 & 483.67 & 4474 & 0 \\
\hline 7 & 26.1 & 84 & 19 \\
\hline 10 & 241.56 & 167 & 9 \\
\hline 12 & 249.19 & 238 & 145 \\
\hline 14 & 176.55 & 633 & 10 \\
\hline 16 & 134.95 & 283 & 13 \\
\hline 25 & 346.3 & 200 & 91 \\
\hline 27 & 661.57 & 254 & 51 \\
\hline 29 & 94.38 & 967 & 47 \\
\hline 31 & 149.24 & 1749 & 0 \\
\hline 33 & 175.55 & 409 & 7 \\
\hline 45 & 393.41 & 5687 & 18 \\
\hline 47 & 252.66 & 5089 & 54 \\
\hline 49 & 577.7 & 722 & 0 \\
\hline 77 & 83.92 & 1402 & 8 \\
\hline 79 & 267.61 & 814 & 200 \\
\hline 81 & 94.74 & 438 & 83 \\
\hline 107 & 101.89 & 216 & 0 \\
\hline 109 & 173.82 & 1068 & 149 \\
\hline 111 & 93.89 & 305 & 12 \\
\hline 113 & 272.47 & 396 & 99 \\
\hline Total & 5353.58 & 25624 & 1062 \\
\hline
\end{tabular}




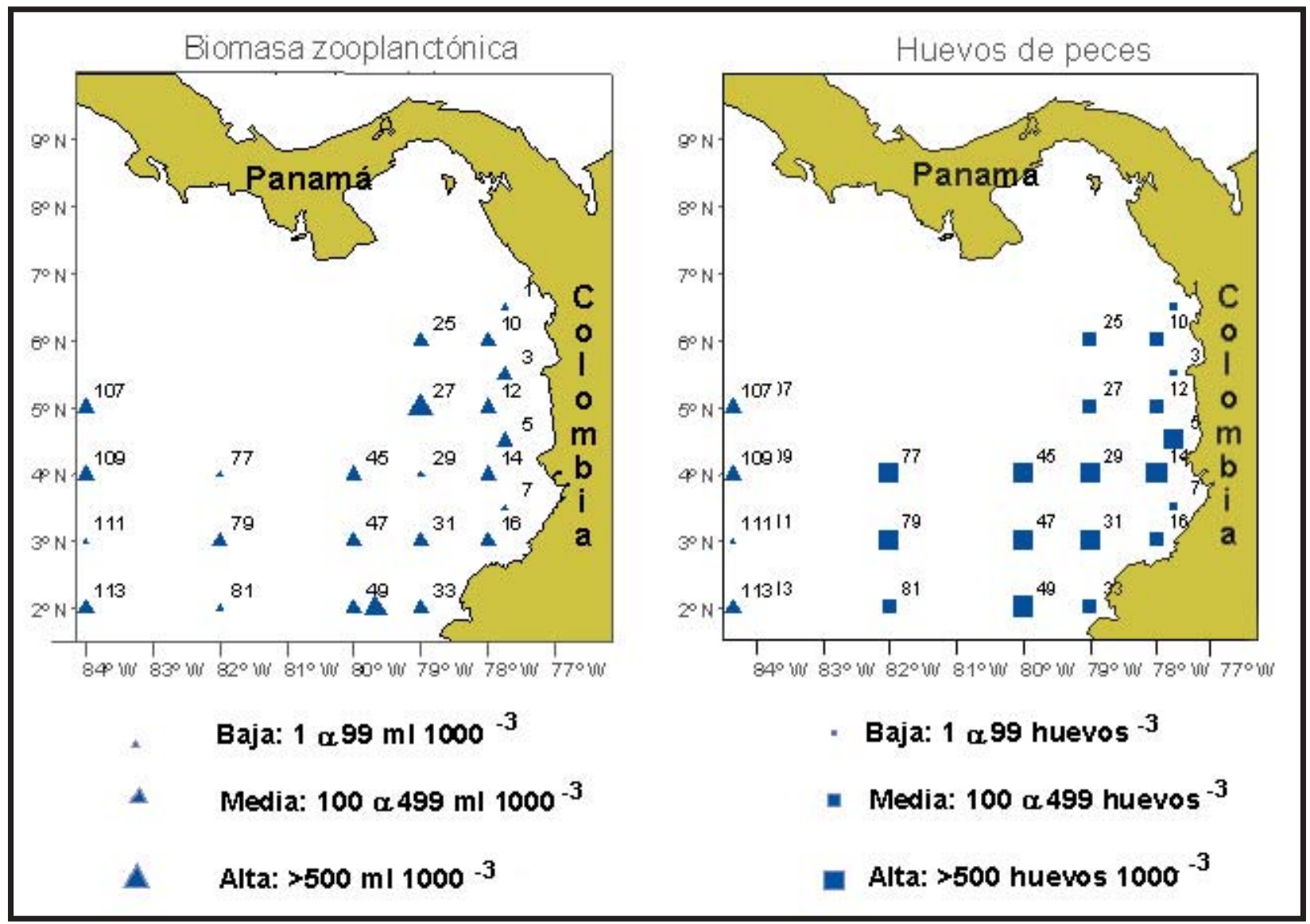

Figura 3. Distribución y abundancia de la biomasa zooplanctónica ( $\mathrm{ml} 1000 \mathrm{~m}^{-3}$ ) y huevos de peces (huevo $1000 \mathrm{~m}^{-3}$ ) en la CPC, durante septiembre2003.

Se identificaron los estadios larvales de 19 especies pertenecientes a 18 familias (Tabla III). Se destacaron por su abundancia y frecuencia de ocurrencia Vinciguerria lucetia (Familia Photichthydae), Hyporhamphus sp. y Oxyporhamphus sp (Familia Hemiramphidae) y Cetengraulis mysticetus (Familia Engraulidae), con una abundancia relativa por familia de 18,15 y $12 \%$, respectivamente. Las larvas de Cyclothone acclinidens (Familia Gonostomatidae) fueron registradas con una abundancia relativa del $11 \%$, aunque ha sido pocas veces detectada en la zona de estudio. La abundancia relativa de las especies pertenecientes a la familia Mycthophidae (Diogenichthys laternatus, Mycctophum aurolacteernatum, Lampanyctus sp., Gonichtys sp) fue del $7 \%$, con una distribución espacial aleatoria. En este mismo sentido, larvas de Mugil cephalus (familia Mughilidae) fueron detectadas en las estaciones costeras, desde Bahía Solano hasta el Sur de la bahía de Buenaventura; mientras que las especies de la familia Carangidae (Chloroscombrus orqueta, C. caranx, C. caballus y Decapterus sp.) sólo fueron detectadas en una estación costera (Figura 4).

Las familias Scombridae y Pomacentridae representaron el 2 \% de la composición porcentual del ictioplancton en la zona de estudio. El $10 \%$ del total de las larvas colectadas, estuvieron representadas por las familias: Gobiidae, Paralichthyidae, Cynoglossidae, Coryphaenidae y Melanostomidae, detectadas en la zona costera; y por las familias Scorpaenidae, Gempylidae, Oneirodidae y Melamphidae, detectadas en la zona oceánica siendo las menos abundantes y de menor ocurrencia en este estudio. 
Tabla III. Abundancia de las familias de larvas de peces (larvas $1000 \mathrm{~m}^{-3}$ ) encontradas en cada una de las estaciones de muestreo durante la campaña oceanográfica Pacífico XXXVIII-ERFEN XXXVI.

\begin{tabular}{|c|c|c|c|c|c|c|c|c|c|c|c|c|c|c|c|c|c|c|c|c|c|c|c|c|}
\hline Estac & 1 & 3 & 5 & 7 & 10 & 12 & 14 & 16 & 25 & 27 & 29 & 31 & 33 & 45 & 47 & 49 & 77 & 79 & 81 & 107 & 109 & 111 & 113 & TOTAL \\
\hline Hem & 8 & 0 & 0 & 7 & 0 & 0 & 10 & 0 & 0 & 0 & 0 & 0 & 0 & c & 0 & 0 & 8 & 0 & 36 & 0 & 0 & 12 & 5 & 06 \\
\hline lugilidae & 17 & 22 & 0 & 7 & 0 & 10 & 0 & 0 & 0 & 0 & 0 & 0 & 0 & 0 & 0 & 0 & . & 0 & 0 & 0 & 0 & 0 & 0 & 56 \\
\hline combridae & 0 & 0 & 0 & 7 & 0 & 0 & 0 & 0 & 0 & 0 & 0 & 0 & 0 & 0 & 0 & 0 & . & 0 & 12 & 0 & 0 & 0 & 0 & 19 \\
\hline ngraulidae & 0 & 0 & 0 & 0 & 0 & 104 & 0 & 0 & 0 & 0 & 0 & 0 & 0 & 0 & 0 & 0 & . & 0 & 0 & 0 & 0 & 0 & 0 & 104 \\
\hline Sonostomatidae & 0 & 0 & 0 & 0 & 0 & 10 & 0 & 0 & 0 & 0 & 0 & 0 & 0 & 0 & 36 & 0 & 0 & 22 & 0 & 0 & 74 & 0 & 12 & 154 \\
\hline Sobiidae & 0 & 0 & 0 & 0 & 0 & 0 & 0 & 13 & 0 & 0 & 0 & 0 & 0 & 0 & 0 & 0 & 0 & 0 & 0 & 0 & 0 & 0 & 0 & 13 \\
\hline aralichthyidae & 0 & 0 & 0 & 0 & 0 & 0 & 0 & 0 & 9 & 0 & 0 & 0 & 0 & 0 & 0 & 0 & 0 & 0 & 0 & 0 & 0 & 0 & 0 & 9 \\
\hline ynoglossidae & 0 & 0 & 0 & 0 & 0 & 0 & 0 & 0 & 9 & 0 & 0 & 0 & 0 & 0 & 0 & 0 & 0 & 0 & 0 & 0 & 0 & 0 & 0 & 9 \\
\hline Carangidae & 0 & 0 & 0 & 0 & 0 & 0 & 0 & 0 & 46 & 0 & 0 & 0 & 0 & 0 & 0 & 0 & 0 & 0 & 0 & 0 & 0 & 0 & 0 & 46 \\
\hline Myctoph & 0 & 0 & 0 & 0 & 0 & 0 & 0 & 0 & 0 & 25 & 0 & 0 & 0 & 0 & 0 & 0 & 0 & 56 & 0 & c & 0 & 0 & 0 & 81 \\
\hline Melanostomidae & 0 & 0 & 0 & 0 & 0 & 0 & 0 & 0 & 0 & 0 & 24 & 0 & 0 & 0 & 0 & 0 & 0 & 0 & 0 & 0 & 0 & 0 & 0 & 24 \\
\hline omacentridae & 0 & 0 & 0 & 0 & 0 & 0 & 0 & 0 & 0 & 0 & 0 & 0 & 7 & 0 & 0 & 0 & 0 & 0 & 0 & 0 & 25 & 0 & 0 & 32 \\
\hline hotichthyidae & 0 & 0 & 0 & 0 & 0 & 0 & 0 & 0 & 0 & 0 & 0 & 0 & 0 & 18 & 0 & 0 & 0 & 112 & 24 & 0 & 0 & 0 & 25 & 179 \\
\hline Gempylidae & 0 & 0 & 0 & 0 & 0 & 0 & 0 & 0 & 0 & 0 & 0 & 0 & 0 & 0 & 0 & 0 & 0 & 11 & 0 & 0 & 0 & 0 & 0 & 11 \\
\hline Oneirodidae & 0 & 0 & 0 & 0 & 0 & 0 & 0 & 0 & 0 & 0 & 0 & 0 & 0 & 0 & 0 & 0 & 0 & 0 & 0 & 0 & 25 & 0 & 0 & 25 \\
\hline Melamphidae & 0 & 0 & 0 & 0 & 0 & 0 & 0 & 0 & 0 & 0 & 0 & 0 & 0 & 0 & 0 & 0 & 0 & 0 & 0 & 0 & 0 & 0 & 12 & 12 \\
\hline Otros & 0 & 0 & 0 & 0 & 9 & 21 & 0 & 0 & 0 & 25 & 0 & 0 & 0 & 0 & 0 & 0 & 0 & 0 & 12 & 0 & 25 & 0 & 25 & 117 \\
\hline Sin identificar & 0 & 0 & 0 & 0 & 0 & 0 & 0 & 0 & 18 & 0 & 0 & 0 & 0 & 0 & 18 & 0 & 0 & 0 & 0 & 0 & 0 & 0 & 0 & 36 \\
\hline TOTAL & 25 & 22 & 0 & 19 & 9 & 145 & 10 & 13 & 91 & 51 & 47 & 0 & 7 & 18 & 54 & 0 & 8 & 200 & 83 & 0 & 149 & 12 & 99 & 1062 \\
\hline
\end{tabular}



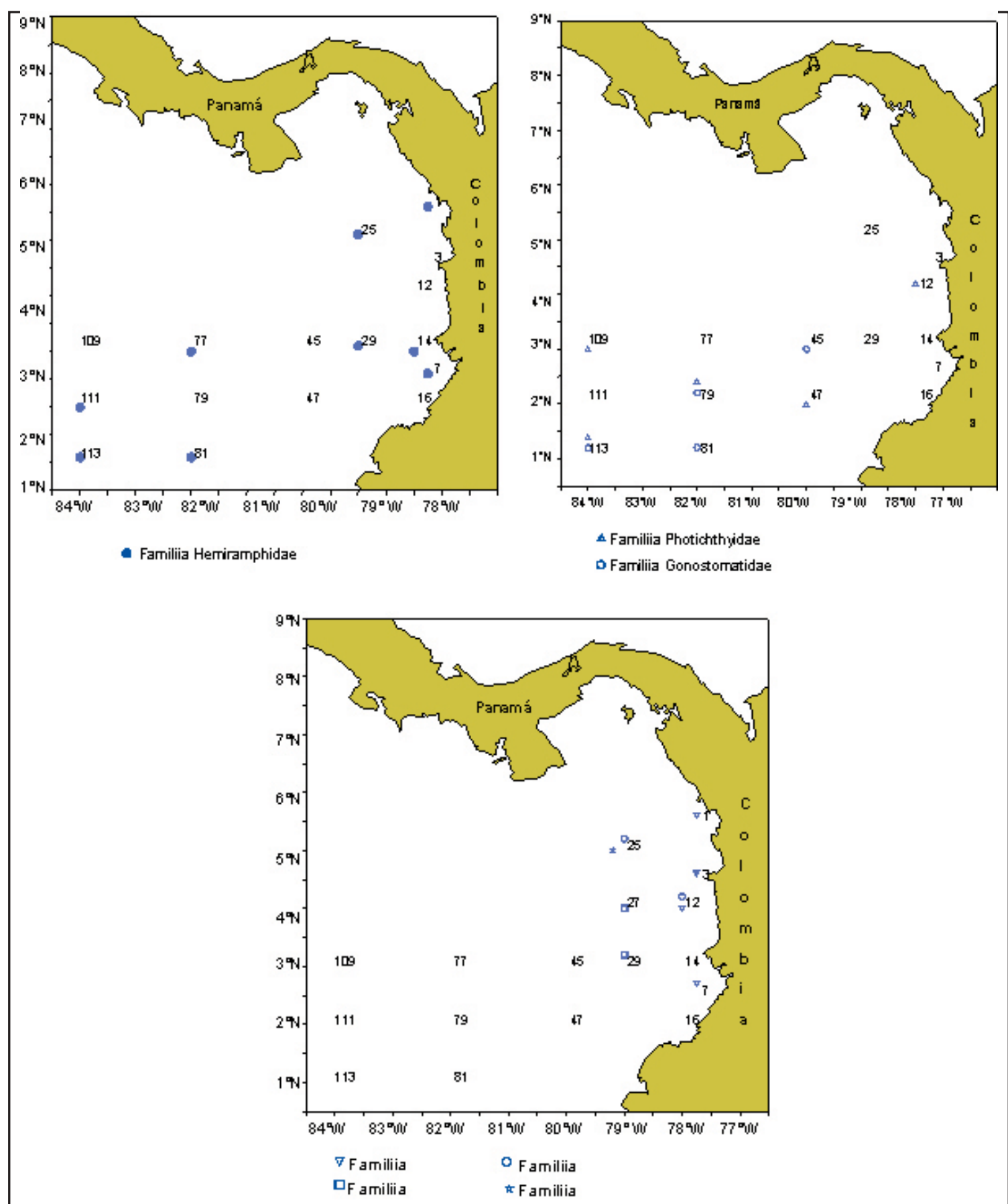

Figura 4. Distribución de las familias de larvas de peces más abundantes en la CPC, durante septiembre 2003.

También fueron identificadas larvas de peces de Citharichthys platophrys (Familia Paralichthyidae), Symphurus sp. (Familia Melanostomidae), Bathophilus filifer (Familia Oneirodidae), Melanphaes $s p$
(Familia Melamphidae) y Coryphaena hippurus (Familia Coryphaenidae), colectadas solamente en una estación oceánica haciendo parte también del $10 \%$ de las familias menos abundantes (Figura 5). 


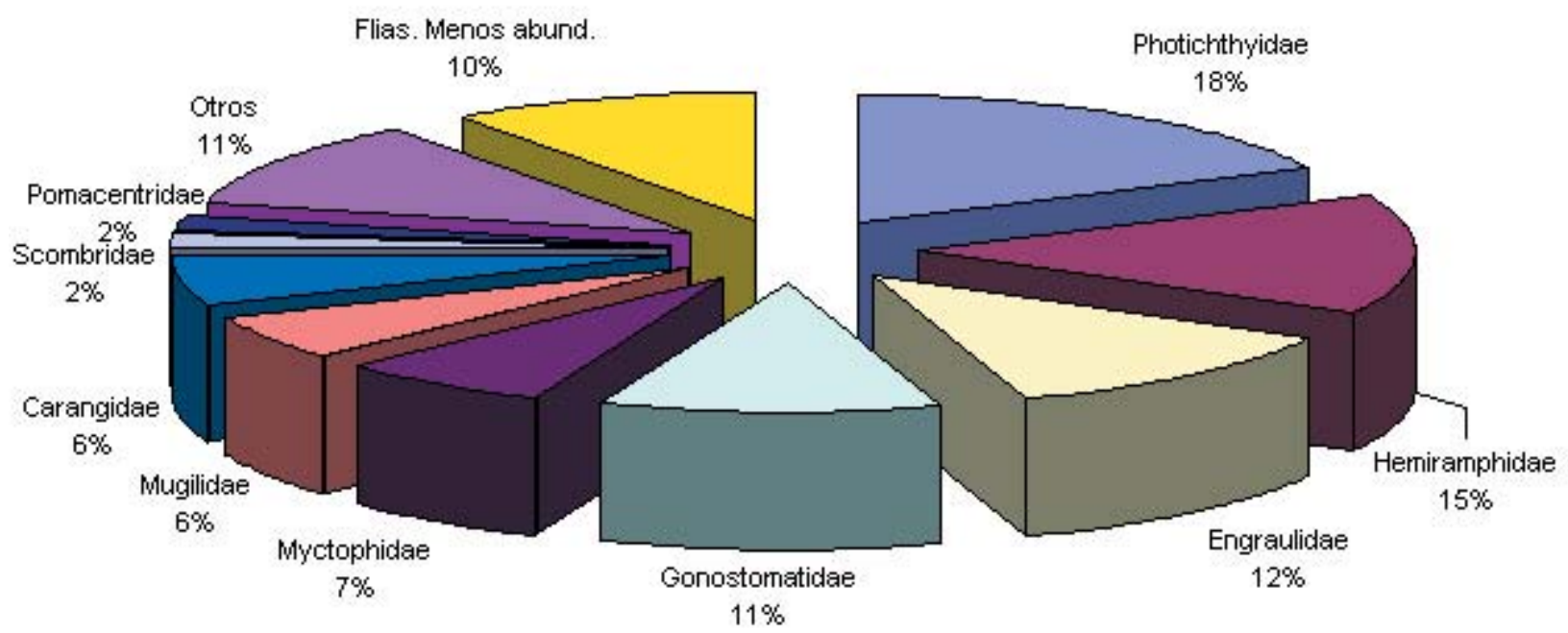

Figura 5. Composición y abundancia porcentual del ictioplancton en la CPC, durante septiembre de 2003.

\section{DisCusión}

La distribución espacial de los parámetros fisicoquímicos durante el período de estudio correspondió a las condiciones oceanográficas estándar definidas por el Centro Control de Contaminación del Pacífico (2002). Se registraron los valores máximos de temperatura en la zona costera, mientras que los valores mínimos se registraron para la zona oceánica. Este patrón de dispersión térmica puede ser consecuencia de la influencia de aguas cálidas provenientes de la contracorriente Ecuatorial, CCE, que se desplaza sobre la región Ecuatorial con dirección Este entre los $5^{\circ} \mathrm{N}$ y $7^{\circ} \mathrm{N}$ hasta los $85^{\circ} 0$ de longitud (CCCP, 2003).

Es importante considerar que durante el período de muestreo (del 2 al 21 de septiembre) la región Norte del área de estudio se encuentra bajo el influjo de las aguas cálidas provenientes de la corriente de Panamá, que impulsadas por los vientos costeros sobre el istmo generan un movimiento ciclónico que caracteriza el patrón general de la circulación superficial de esta región del Panama Bigth. Además, los vientos alisios del Sur favorecen la intrusión de aguas frías del suroeste entre $82^{\circ} 0$ a $84^{\circ} 0$, provenientes del sistema de circulación de la corriente de Humboldt (Wyrtki 1965; CCCP, 2003).
Durante el período de estudio se identificó una fuerte isohalina que dividió la CPC en sentido longitudinal en una zona costera, con valores de salinidad bajos, y una región oceánica, con registros de salinidad más altos. Esta división halina tan característica, probablemente sea una respuesta directa al incremento del aporte fluvial en la zona costera durante la época principal de lluvia en la región, como lo indica Forsbergh 1969.

En este mismo sentido el patrón de dispersión espacial de la concentración de oxígeno disuelto sugiere que las más altas concentraciones registradas en la zona oceánica son consecuencia directa de la presencia de aguas superficiales tropicales, provenientes del Pacífico Central. La poca variabilidad en el patrón de distribución espacial de la clorofila- $a$ es una característica típica de las aguas oceánicas superficiales tropicales, debido a la baja concentración de nutrientes. Sin embargo, el registro de valores superficiales de clorofila- $a$ relativamente altos en la zona costera, es una respuesta directa al aporte de nutrientes a través del drenaje de agua dulce de la vertiente oriental de la cordillera Occidental.

Los valores estimados de biomasa zooplanctónica en la CPC durante septiembre pueden ser considerados como valores medios para 
la zona de estudio. Las concentraciones más altas de biomasa zooplanctónica se registraron en la zona costera, influenciadas, principalmente, por la topografía del fondo (costa somera) y la abundancia de alimento (biomasa fitoplanctónica estimada como concentración de clorofila- $a$ ). Aunque en la zona costera de la CPC no se han registrado zonas de surgencia, las condiciones geomorfológicas locales predominantes como: cabos, bahías y estuarios, probablemente, favorecen el desarrollo de agregaciones espaciadas de fitoplancton y zooplancton que incrementan los valores de biomasa.

Debido a que los huevos son derivadores pasivos, es de esperar una alta frecuencia de ocurrencia de estos elementos en la zona de estudio y una asociación directa entre su patrón de dispersión espacial y el patrón de circulación superficial (LópezPeralta, 1984). En este sentido, la corriente de deriva costera o corriente Colombia es más intensa durante el mes de septiembre como consecuencia del incremento en la fuerza del sistema de vientos Alisios del Sur, lo que favorece el transporte pasivo de los huevos hacia afuera de la costa explicando su ocurrencia en la zona oceánica. Aunque la presencia de larvas de peces estuvo estrechamente asociada con la presencia de huevos de peces, su abundancia relativa fue baja llegando incluso a no registrarse larvas de peces en algunas de las estaciones debido, probablemente, a que el tipo de red utilizado durante la pesca de zooplancton (cónica simple), la forma del arrastre (superficial) y la hora del muestreo (generalmente diurno) no son los óptimos para este tipo de estudio.

Como la gran mayoría de los organismos planctónicos, las larvas de peces efectúan, en escalas temporales circadianas, migraciones verticales a lo largo de la columna de agua, de tal forma que durante el día permanecen a media agua y durante la noche se agrupan en la capa superficial. Esto hace que se introduzcan errores de interpretación por falta de muestreos nocturnos ya que la mayoría de la toma de muestras se hizo en horas del día. Además, el herraje estándar de una red cónica simple incrementa las fuerzas hidrodinámicas durante el arrastre, lo que favorece la evasión de las larvas de peces a la acción filtrante de la red.

Aunque la asociación entre la dispersión espacial de las larvas de peces y la distribución espacial de los parámetros fisicoquímicos evaluados no fue clara, la familia Hemiramphidae exhibió la mayor frecuencia de ocurrencia en el área de estudio; mientras que la familia Potichthyidae presentó la mayor abundancia con una distribución no uniforme, y la familia Engraulidae, tercera en orden de abundancia, se encontró principalmente asociada a zonas costeras de menor salinidad.

De las familias y especies de larvas de peces registradas en el presente estudio, las familias Mugilidae (Mugil cephalus), Engraulidae (Cetengraulis mysticetus) y las especies de la familia Carangidae pueden ser consideradas como de importancia comercial. Sin embargo, las frecuencias de ocurrencia y abundancia establecidas para éstas no fueron relevantes en el área de estudio.

Considerando las relaciones tróficas de las comunidades pelágicas, es de esperar la ocurrencia de relaciones directas o indirectas entre la concertación de clorofila- $a$ y la abundancia del ictioplancton en una región específica (Ciechomski, 1981). Sin embargo, en el presente estudio no fue posible evaluar adecuadamente el grado de asociación entre estas dos variables. Además, debido a la baja resolución espacial y temporal del muestreo, no fue posible establecer alguna tendencia de agregación para definir áreas y/o épocas de desove o reclutamiento de peces. A pesar de que el tipo de aproximación metodológica utilizada durante el presente estudio no permite evaluar la biomasa esperada de poblaciones de peces futuras, la información derivada incrementa el conocimiento general de la comunidad ictioplanctónica de esta región poco explorada del Pacífico Oriental Tropical.

\section{Conclusiones}

El análisis espacial de los parámetros físicoquímicos-biológicos sugiere que las condiciones oceanográficas en la zona de estudio se consideran normales de acuerdo con los estándares establecidos por el CCCP.

En relación con la composición del ictioplancton para la CPC estuvo representado por altas y medias concentraciones de huevos, y por bajas concentraciones de larvas de peces.

La familia de larvas de peces con más amplia distribución en el área de estudio fue Hemiramphidae, mientras que la familia Photichthyidae fue la de mayor abundancia. 
En las estaciones 25 y 113 se registraron los mayores valores de diversidad y riqueza ictioplanctónicas. Sin embargo, el tipo de red y arrastre utilizado durante la pesca de zooplancton tienden a sesgar la muestra de ictioplancton, considerando la migración vertical que esta presenta. Por tanto, se recomienda para posteriores trabajos realizar arrastres oblicuos con red Bongo.

\section{Agradecimientos}

A la Dirección General Marítima, DIMAR; al Centro Control Contaminación del Pacífico, CCCP; a la Unidad Administrativa Especial del Sistema de Parques Nacionales Naturales, UAESPNN, y al Departamento de Biología de la Universidad del Valle por el apoyo y financiación de presente estudio.

A la Universidad del Valle por el aporte de equipos e instalaciones.

A Luis Alfonso Zapata por su ayuda en la revisión de este artículo, al Capitán de Fragata Ernesto Durán y su tripulación, por el apoyo logístico a bordo del buque oceanográfico ARC Malpelo.

Al Suboficial Segundo Yuber Alberto Paul Cantor, por su orientación y explicación en la elaboración de mapas.

\section{Literatura Citada}

Ahlstrom, E. 1983. Ontogeny and systematic of fishes. Based on An international Symposium dedicated to the memory of Elbert Halvor Ahlstrom. La Jolla California. Tomo I, II. 760 pp.

Beltrán-León, B. 1992. Identificación y descripción de los tipos de huevos, larvas y postlarvas de Engraulidos y Clupeidos (Pisces, Clupeiformes) en el Pacífico colombiano - febreromarzo de 1991. Santiago de Cali, 134 pp. Trabajo de grado (Biología Marina). Departamento de Biología Marina, Facultad de Ciencias, Universidad del Valle.

Beltrán-León B., G. Ramos, J. Escobar y J. Tovar. 1994. Distribución y abundancia de huevos y larvas de Opisthonema spp. (Gill, 1861) y Cetengraulis mysticetus (Gunther, 1866) (Pisces: Clupeiformes) en el Pacífico colombiano, durante enero de 1993. Boletín Científico INPA, (2): 181-199 pp.
Beltrán-León, B. y Ríos. 2000. Estadios tempranos de peces del Pacífico colombiano. Instituto Nacional de Pesca y Acuicultura INPA. Buenaventura. 727 pp.

CCCP. 2002. Compilación Oceanográfica de la Cuenca Pacífica Colombiana. San Andrés de Tumaco: Centro Control Contaminación del Pacífico, Serie Publicaciones Especiales Vol. 1, 109 pp.

CCCP. 2003. Estudios de los Eventos ENOS en la Cuenca del Pacífico Colombiano durante el 2003. Informe técnico. San Andrés de Tumaco. 51 pp.

Ciechomski, J. 1981. Ictioplancton. En: Atlas del zooplancton del Atlántico Sudoccidental y métodos de trabajo con zooplancton marino. Edit. D. Boltovskoy. Publ. Espec. INDENEP. Mar de Plata, Argentina. Pp. 829-861.

Echeverri, C. y H. Bergamín. 1982. Estudio preliminar de taxonomía y distribución de larvas de peces en la bahía de Buenaventura. Santiago de Cali, 99 pp. Trabajo de grado (Biología Marina). Departamento de Biología Marina, Facultad de Ciencias, Universidad del Valle.

Forsbergh, E. 1969. Estudio sobre la Climatología, Oceanografía y Pesquería del Panamá Bight. CIAT. Boletín, La Jolla, California, Vol. 14 (2): 146-385.

Jiménez, S. 1991. Contribución al estudio de larvas y huevos de peces para la costa Pacífica colombiana. Crucero Pacífico XIII EFRÉN X, noviembre de 1988. Santiago de Cali, 99 pp. Trabajo de grado (Biología Marina). Departamento de Biología Marina, Facultad de Ciencias, Universidad del Valle.

López-Peralta, R. 1984. Descripción y abundancia del ictioplancton entre 0 y $10 \mathrm{~m}$, de profundidad para el Pacífico colombiano (Nov. 1982). Bogotá. Trabajo de grado (Biólogo Marino), 218 pp. Facultad de Biología Marina, Universidad Jorge Tadeo Lozano.

Montagut, C. y M. Castillo. 1989. Distribución de los dinoflagelados durante los monitoreos del programa ERFEN en el Pacífico colombiano. Crucero Pacífico XI - ERFEN VIII (Nov. 1987), Crucero Pacífico XII- ERFEN IV (Mar. 1988). Tesis de grado (Biología Marina), 186 pp. Facultad de Bilogía Marina, Universidad Jorge Tadeo Lozano.

Moreno, A. 1995. Descripción y abundancia de larvas y post-larvas de Scianidos (Pisces: 
Perciformes) en el Pacífico colombiano durante 1991. Santiago de Cali, 199 pp. Trabajo de grado (Biología Marina). Departamento de Biología Marina, Facultad de Ciencias, Universidad del Valle.

Moser, H. 1996. The early stages of fishes in the California corrent region. Cal. Coop Ocean. Fish. Inv. Atlas. 1441 pp.

Rueda-Montenegro, C. y P. Caraballo. 1984. Composición distribución y abundancia del ictioneuston en el Pacífico colombiano. Nov, Dic de 1982. Bogotá, 199 pp. Tesis de grado (Biología Marina). Facultad de Biología Marina, Universidad Jorge Tadeo Lozano.

Rueda-Montenegro, C. y B. Beltrán-León. 1992. Ictioplancton de las familias Clupeidae y Engraulidae en el Litoral Pacífico colombiano, durante 1991. Memorias del VIII Seminario Nacional de Ciencias y Tecnológicas del Mar y Congreso Centroamericano y del Caribe de Ciencias del Mar. Santa Marta. Pp. 735-743.

Wyrtki, K. 1965. Corrientes Superficiales del Océano Pacífico Oriental Tropical. CIAT. California, (15): 295-303. 